\title{
Unified Traceability Information System of Logistics Pallet Based on the Internet Of Things
}

\author{
LIN Xing-zhi \\ Guangxi Economic Management Cadre College,Nanning,China \\ Ixz4562509@139.com
}

Keywords: logistics; Internet of things (IOT); unified information; intelligent tracing system

\begin{abstract}
The paper focuses on the intelligent logistics pallet, networking, traceability information technology and unified logistics information system, and puts forward unified traceability information system of intelligent logistics pallet based on the Internet of things (IOT), displaying innovative system realization method and technical system. IOT intelligent logistic pallet traceability system is an integrated fusion application of RFID, GIS, GPS, computer telecommunication integration together with Internet technology; in system design and implementation, TOT and RFID coupling mechanism, CTI and API interface programming are adopted for construction of three intelligent system service architecture of logistics pallet traceability system including application, business and physics; in the logistics pallet regular operation, reading and writing RFID equipments, labels and telecommunications network, Internet, and other unified information communication mode are fused into the intelligent logistics information system, so as to realize the intelligent logistics pallet identity identification and traceability function. The system test output results indicate that, intelligent logistics pallet unified information system is equipped with intelligent recognition, management and tracing function and business process reengineering capacity including production promotion, distribution, transport logistics and allocation.
\end{abstract}

\section{Introduction}

A unified logistics information traceability system is the PCV-I link of the system service in each business stage of logistics supply chain, i.e. data preparation, collection, verification and identification and a series of fusion structure of tracing mechanism and integrated application [1]. Radio Frequency Identification (RFID) logistics pallet plays roles of "fountainhead of the third profit" and logistics transparent management in the traceability system; it is a typical application of Internet of things (IOT). RFID pallet is a plate which is provided with identification (ID tag) and is used to carry goods for cargo handling, transport, storage and distribution and for loading and unloading of forklift; it is the logistics industry's "mobile platform" and "moving ground". Logistics traceability system pallet can record the ownership, bearing capacity, transport condition, objective area and other traceability information and process operation state [2]. For the logistics business process, the whole supply chain involves many factors related with the pallet transportation and articles, so the system is an integrated application with Geographical Information System (GIS), Global Positioning System (GPS), Computer Telecommunication Integration (CTI) and Internet, forming an interactive ubiquitous link mechanism in a distributed logistics system, in order to realize the intelligent recognition, positioning, tracking, monitoring and management in logistics processes, so that the users can easily access logistics information query and management with mobile phone, PDA or Internet [3].

\section{IOT-based construction of unified information traceability system of logistics pallet}

IOT-based unified information traceability system of logistics pallet is adopted in the supply chain management, covering the upper pallet carriers and downstream enterprises of manufacturing, processing, sales, retail and transportation, and forming the regular pallet operation system [4]. RFID equipments consist of RFID pallet label, reader-writer; unified information communication mode and 
tools are mainly CTI server and Internet Short Messages (SMS) Gateway (ISMG) and GSM modem, and it can be developed based on the API, and can be easily adopted on WEB, mobile phone and PDA. The unified logistics information system can have automatic tracing, identification and provide optimal allocation schemes for transportation, warehousing, production for the RFID pallet. The system consists of layers of physics, business and application, and the structure is shown in Figure 1.

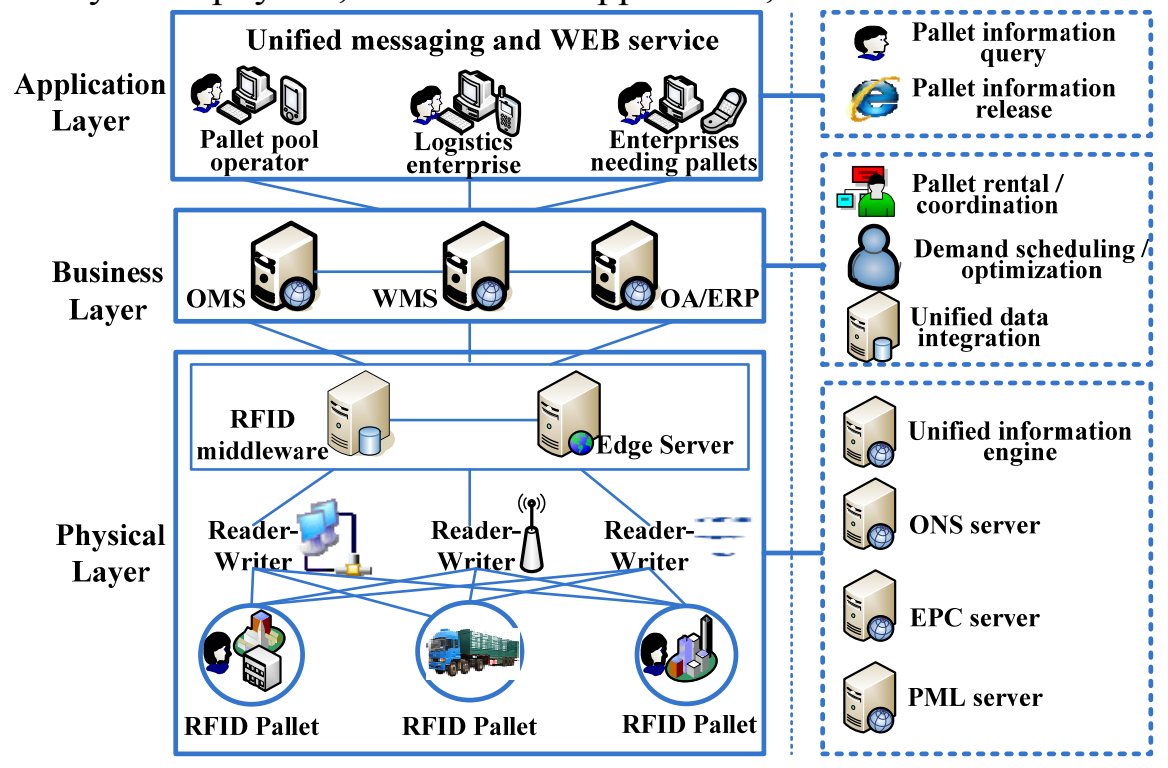

Figure 1. Structure of logistics pallet traceability system

The physical layer is constituted by the pallet RFID tag, reader-writer, wired or wireless radio frequency equipment, RFID middleware and the edge servers (ES) etc., forming a based network of tracing. When the reader-writer receives the command, it can generate magnetic field, and the RFID pallet having received signal will, relying on the energy from induction current, transfer the ID, specifications, goods name, quantity and weight, stored in RFID to the business layer; then the data analysis system of business layer, Operations Management System (OMS) will integrate with physical layer's edge server, Object Name Service (ONS ), Electronic Product Code Service (EPC), Physical Markup Language Service (PML), and then, through filter, correlation and aggregation, radio frequency middleware will carry out real-time information processing of the pallet information in supply chain nodes and pallet storage location, recycling pallet status. Application layer will transform the traceability data from the pallet unified information system into a unified data representation format, providing service by means of WEB, mobile phone Internet and SMS and other forms.

\section{System design and Implementation}

Consistent pallet operation and RFID information tracing. Pallet is the most foundational and important goods unit in supply chain, and has been widely used in logistics supply chain links including production, storage and retail [5]. In the traceability system, the ITO technology is mainly used for intelligent products traceability and product configuration process: (1) input RFID pallet UID, attribution, bearing capacity, specifications and other information, flexibly choose EPC coding standard; (2) write the article information on pallet RFID label, including the weight, quality, quantity, suitable temperature, humidity, transporter, destination, receiver; (3) have storage, change or assemble fragmented containers; re-associate RFID data and labels; (4) with GSM and other communication means, collect, identify and trace pallet information, and form consistent logistics pallet operation and business process reengineering mechanism, so as to realize fast and accurate inventory, transfer, storage, transportation, production, leasing, and improve overall transparency of enterprise logistics.

RFID has two ways, electromagnetic induction or electromagnetic propagation, consisting of RFID tags and reader-writer; it achieves non-contact reading, writing and electronic identification tag. 
The RFID tags on the pallet is environment-resistant, namely strong waterproof, oil-resistant and chemical-resistant, and can repeatedly override the RFID tag data; traceability signal magnetic field has a strong ability of penetrating and diffraction, and the RFID magnetic field induction and responses will not be affected by the non-metallic coating of articles.

In the consistent logistics pallet operation system, in order to address different pallet operations and information traceability, various RFID coupling mechanism and standard are adopted in necessary. RFID frequency includes microwave $(2.45 \mathrm{GHz})$, ultra high frequency $(860-960 \mathrm{MHz}$, $433 \mathrm{MHz})$, high frequency $(13.56 \mathrm{MHz})$, low frequency $(125 \mathrm{KHz})$, and generally $860-960 \mathrm{MHz}$ and $2.45 \mathrm{GHz}$ is used in the pallet, to meet the transmission performance and automation operation. The power supply is realized in two forms: internal active power and passive electromagnetic induction, and due to strong mobility of pallet, the latter is generally used. Information collection traced by RFID is read in group, that is to say, pallet tag information can be read wholly.

Unified information realization. In order to realize the IOT-based pallet traceability system of unified information, hardware API programming interface is developed taking industrial GSMmodem as an example, the system is realized with the help of the B/S framework and ASP programming; it can be use on WEB, mobile phone text messages and other communication means. GSMmodem is composed by SIEMENS or WAVECOM as the core, connected to the server through the serial port; it controls system operation and RFID equipment by AT commands [6]. Part of the procedures is as follows:

Initialize and connect to GSMmodem, obtain dynamic library dllgsm.dll function, device identifies communication port, setting system will automatically detect ( NULL ), baudrate identification system will automatically detect baud rate ( NULL ), initstring identifies the initialization command (NULL), charset identifies the communication character set (NULL), swHand shake identifies whether the software handshake (FALSE):

[DllImport("dllgsm.dll",

EntryPoint = "MODEMInitNew",

CharSet $=$ CharSet.Ansi,

CallingConvention $=$ CallingConvention.StdCall)]

public static extern bool MODEMInitNew(

Set SMS sending mode, service Center Address identifies the number of message center (NULL), encodeval identifies SMS coding format (8 means Chinese SMS coding), text identifies the message content, textlen identifies the message length, TEL number identifies the phone number receiving pallet tracing instruction message, requestStatusReport identifies the status report. The following is the code:

[DllImport("dllgsm.dll", EntryPoint = "MODEMSMSsend",

CharSet $=$ CharSet.Ansi,

CallingConvention $=$ CallingConvention.StdCall)]

public static extern bool MODEMSMSsend(

string serviceCenterAddress,

Set the received SMS string as the following format: mobile phone number-SMS content-pallet traceability code; in the information format, form pallet back instruction and information collection mechanism. The code is:

[DllImport("dllgsm.dll",

EntryPoint = "MODEMSMSReadAll",

CharSet $=$ CharSet.Ansi,

CallingConvention $=$ CallingConvention.StdCall)]

public static extern string MODEMSMSReadAll(int RT_opt);

Import namespace using System.Text, when the page loads, in the GMS class, the MODEMGetSnInfoNew method, MODEMGetDevice method and MODEMGetBaudrate method 
are specifically used to display the machine number (Sn), COM port and BTL. The specific codes are as follows:

protected void Page_Load(object sender, EventArgs e)

\{

this.txtJQHM.Text= Basic.GMS.MODEMGetSnInfoNew(txtCOM.Text, txtBTL.Text);

this.txtCOM.Text= Basic.GMS.MODEMGetDevice();

\}

this.txtBTL.Text= Basic.GMS.MODEMGetBaudrate();

The devices are connected for SMS sending, through the MODEMInitNew method in GMS class, if the connection is right, the program will, through MODEMSMSsend method in GMS, sent the input pallet traceability command message to the specified RFID reader-writer, while if the connection fails, the program will output error information through GMS's MODEMGetErrorMsg method:

Protected void btnSend_Click(object sender,EventArgs e)

\{

$$
\text { if }(\text { txtSJHM.Text }==" ')
$$

Page.RegisterStartupScript("',"<script>alert(' Phone number can't for empty! ')</script> "); txtSJHM.Focus();

return;

If (Basic.GMS.MODEMSMSsend(null, 8, txtContent.Text, Encoding.Default.GetByteCount(txtContent.Text), txtSJHM.Text, false) $==$ true)

Page.RegisterStartupScript("'," <script>alert(' Send success!')</script>"); else

Page.RegisterStartupScript("'","<script>alert('

Send

failure!'"

Basic.GMS.MODEMGetErrorMsg()+"')</script $>$ ");

The program receiving SMS will, through the MODEMSMSReadAll method in GSM class, read the pallet traceability collection SMS sent to the mobile phone card:

protected void btnResvice_Click(object sender, EventArgs e)

txtContent.Text= Basic.GMS.MODEMSMSReadAll(1);

if (txtContent.Text.Length $<=0$ )

\{

Page.RegisterStartupScript("',,"<script>alert(' Empty information ! ')</script> ");

return;

\}

txtSJHM.Text = txtContent.Text.Substring $(0,13)$;

txtContent.Text $=$ txtContent.Text.Substring(13, txtContent.Text.Length -13$)$;

\}

\section{Output of the test result}

IOT-based unified information traceability system of logistics pallet is tested in business application by means of manual tracing and RFID unified information traceability, with testing tools of telephone, mobile phone, PAD, Internet and RFID logistics pallet, RFID reader-writer. The RFID tracing test indicates that, users can not only have real-time automatic information tracking in the webpage or mobile phone system, also can realize digital map position and trajectory display, which is starkly contrasted with manual traceability. Test output is shown in Table 1:

Table 1 The output of system test results 


\begin{tabular}{lllll}
\hline Test mode & Query mode & Time consuming & Accuracy & $\begin{array}{l}\text { Real-tim } \\
\text { e }\end{array}$ \\
\hline Manual traceability & $\begin{array}{l}\text { Telephone/IM } \\
\text { Automatic traceability } \\
\text { GSM/WEB }\end{array}$ & $\begin{array}{l}\text { Long, uncertain } \\
\text { Read speed } \\
<1 \mathrm{u}, \mathrm{GSM}<3 \mathrm{~s}\end{array}$ & $\begin{array}{l}\geq 99 \% \\
\text { RF traceability }\end{array}$ & yes \\
\hline
\end{tabular}

The results demonstrate that, in the consistent pallet operations, the traceability in ITO unified information system makes it more likely to realize the logistics business process reengineering, so that the third party logistics and logistics management are more transparent

\section{Conclusions}

IOT-based unified information traceability system of logistics pallet, taking advantage of RFID automatic identification and network technology, designs visualization traceability frame of the entire supply chain information of logistics pallet. On one hand, logistics pallet information and user information communication tool can achieve non-contact interaction and processing, so that the logistics pallet efficient business process reengineering, process and information feedback can be realized, which constitutes a real-sense ITO, therefore, the logistics pallet information can be updated in real time and accurately tracked, to maximize the interests of enterprises and resources effective utilization. The RFID pallet monitoring and in road logistics anomalies can be reflected in real time, the vacancy rate of supply chain is particularly precisely controlled in real time; then the consistent pallet operation and transport configuration optimization is realized to reduce transportation costs and improve service quality. On the other hand, the application fusion of CTI and ITO technology and effective use of WEB, mobile phone and other unified communication traceability means satisfy the users' right to know and to control and improve the benefit of logistics management. The unified logistics information traceability system can promote the organic union of consistent pallet operation and supply chain link, making the integration of logistics, product, information identification and control.

\section{References}

[1] Can Wang.The Traceable System in Agricultural Logistics and its Application[J]. The Journal of Shandong Agricultural Administrators' College,2010,(04):52-53.

[2] XU Qi.Logistics Pallets Pooling Service Supply Chain System and the Optimal Management[J].China Business and Market,2010,(03):22-25.

[3] X.Z LIN. Analysis on Security Strategy of Double-factor Authentication in Unified Logistics Information System [C]. USA: Scientific Research Publishing,2010:421-425.

[4] ZHU Wen-he.Realization of Whole-process Intelligent Supply Chain Distribution Services Based on the Internet of Things[J].Logistics Technology,2010,(13):172-173.

[5] DENG Yi-tao.The Internet of Things Technology Application in the Supply Chain Management[J].Logistics Sci-Tech,2010,(09):92-94.

[6] X.Z LIN. Design and Realization of Concise Unified Logistics Information System[C]. USA: Scientific Research Publish-ing,2010:414-418. 